\title{
Immune checkpoint inhibitor plus tyrosine kinase inhibitor for unresectable hepatocellular carcinoma in the real world
}

\author{
Diyang Xie ${ }^{1 \#}$, Qiman Sun ${ }^{1 \#}$, Xiaoying Wang ${ }^{1}$, Jian Zhou ${ }^{1,2}$, Jia Fan $^{1,2}$, Zhenggang Ren ${ }^{1}$, Qiang Gao \\ ${ }^{1}$ Liver Cancer Institute, Zhongshan Hospital, and Key Laboratory of Carcinogenesis and Cancer Invasion (Ministry of Education), Fudan University, \\ Shanghai, China; ${ }^{2}$ Institute of Biomedical Sciences, Fudan University, Shanghai, China \\ Contributions: (I) Conception and design: Q Gao, X Wang, J Zhou, J Fan, Z Ren; (II) Administrative support: Q Gao, X Wang, J Zhou, J Fan, Z Ren; \\ (III) Provision of study material or patients: All authors; (IV) Collection and assembly of data: D Xie, Q Sun. (V) Data analysis and interpretation: All \\ authors; (VI) Manuscript writing: All authors; (VII) Final approval of manuscript: All authors. \\ "These authors contributed equally to this work. \\ Correspondence to: Qiang Gao, MD, PhD. Department of Liver Surgery and Transplantation, Liver Cancer Institute, Zhongshan Hospital, Fudan \\ University, Shanghai 200032, China. Email: gao.qiang@zs-hospital.sh.cn; Zheng-Gang Ren, MD, PhD. Department of Hepatic Oncology, Liver \\ Cancer Institute, Zhongshan Hospital, Fudan University, Shanghai, China. Email: ren.zhenggang@zs-hospital.sh.cn.
}

Background: This study aimed to evaluate safety and efficacy of programmed death-1 (PD-1) inhibitor sintilimab plus tyrosine kinase inhibitors (TKI) in a real-word cohort of patients with unresectable hepatocellular carcinoma (uHCC).

Methods: A total of 60 patients treated with sintilimab plus TKI between February 2019 and December 2019 were enrolled. Radiological response was recorded by computed tomography (CT) or magnetic resonance imaging (MRI) at baseline and every 6-12 weeks after treatment initiation. Tumor response was assessed according to the Response Evaluation Criteria in Solid Tumors (RECIST) 1.1 and HCC-specific modified RECIST (mRECIST).

Results: As of the data cutoff on September 30st, 2020, the median duration of follow-up was 10.4 (4.3-23.9) months. The objective response rate (ORR) and disease control rate (DCR) were $36.7 \%$ (95\% CI: 24.948.5\%), 81.7\% (95\% CI: 71.9-91.5\%) according to the RECIST 1.1, and 52.8\% (95\% CI: 39.1-66.5\%), 83.0\% (95\% CI: 73.2-93.8\%) according to mRECIST criteria. Among 36 HCC patients with multinodular HCC or locally-advanced HCC with portal vein tumor thrombus (PVTT), 14 patients received one session of transarterial chemoembolization (TACE) within 1 month before or after the combinational systemic therapies, and the rest 22 patients did not receive any local regional therapies. After propensity score matching, patients from the TACE group tended to have a longer PFS (median, 10.1 vs. 9.1 months, $\mathrm{P}=0.73$ ) than those from the non-TACE group but without significant differences. A total of 8 patients received surgical resection after the combined systemic therapies and 3 patients achieved pathological CR. No recurrence or metastasis was observed in 6 patients. A total of $46(76.7 \%)$ patients reported adverse event (AE) with any grade and $8(13.3 \%)$ patients discontinued the combination therapy due to grade $3 / 4$ severe adverse events.

Conclusions: PD-1-targeted immunotherapy sintilimab plus TKIs exhibited promising efficacy with tolerable toxicity in unresectable HCC. The addition of TACE to the combined systemic therapies also resulted in a favorable tumor control and safety. For select responders, surgical resection might be a choice for radical treatment.

Keywords: Hepatocellular carcinoma (HCC); immune checkpoint inhibitor; tyrosine kinase inhibitor (TKI); tumor response

Submitted Oct 21, 2020. Accepted for publication Jan 15, 2021.

doi: $10.21037 /$ atm-20-7037

View this article at: http://dx.doi.org/10.21037/atm-20-7037 


\section{Introduction}

Hepatocellular carcinoma (HCC) is the most common primary liver cancer and the fifth leading cause of cancerrelated deaths worldwide (1). Locoregional therapies (LRT) and surgery remain the optimal treatment for early and intermediate HCC, whereas systemic treatment is recommended for patients at advanced stage. Approximately $50 \%$ of patients receive systemic therapy at some points during the disease course (2). Multi-target tyrosine kinase inhibitor (TKI) sorafenib was the only FDA-approved systemic treatment before 2017 (3). New breakthroughs have been made during the past three years since four other TKIs including lenvatinib, regorafenib, carbozantinib and ramucirumab are added as the first-line or secondline systemic therapies for HCC (4-7). Apatinib, a select VEGFR-2 inhibitor, is indicated as another secondline treatment option in China (8). Immune checkpoint inhibitors (ICIs) including cytotoxic $\mathrm{T}$ lymphocyteassociated protein 4 (CTLA-4) and programmed cell death protein 1 (PD-1) inhibitors, as potential curative treatments for multiple cancers, have also become adopted therapies for advanced HCC. Two PD-1 monoclonal antibodies (mAbs) nivolumab and pembrolizumab have been indicated for HCC patients progressed on sorafenib, according to a durable objective response rate (ORR) of $20 \%$ and $17 \%$ respectively in the phase 2 CheckMate-040 and KEYNOTE-224 studies $(9,10)$. However, the unmet overall survival (OS) benefit in the phase 3 CheckMate-459 and KEYNOTE-240 studies frustrated applying single-agent PD-1 inhibitor in advanced HCC (11). Combination of ICIs with vascular endothelial growth factor (VEGF)-targeted agents has exhibited superiority to monotherapy in many solid tumors (12). In HCC, pembrolizumab in conjunction with lenvatinib showed an ORR of $36.0 \%$ with acceptable toxicity in a phase Ib/II study (13). The result of the phase 3 IMbrave150 study released recently demonstrated that treatment with PD-1 ligand (PD-L1) mAb atezolizumab plus VEGFR mAb bevacizumab was associated with a significantly better overall survival (OS) and progressionfree survival (PFS) than sorafenib in patients with unresectable HCC not previously treated with systemic therapy (14). Sintilimab is another PD-1 mAb with a higher affinity and slower off-rate compared with pembrolizumab and nivolumab (15). It has been available for patients with advanced cancer in China since 2019. Preliminary results of a phase Ib study showed treatment with sintilimab plus VEGF mAb IB305 resulted in an ORR of $33.3 \%$ and a 6-month PFS of $60.5 \%$ in patients with advanced HCC (16). A phase 3 ORIENT-32 study evaluating the efficacy and safety of sintilimab plus IB305 as a first-line treatment for advanced HCC is underway (NCT03794440). For patients with unresectable HCC ineligible for or reluctant to enter the clinical trials, sintilimab has been administered either in combination with TKIs or in addition to resistant TKIs in our center. Transarterial chemoembolization (TACE) and surgical resections were also applied for select patients during the combined systemic therapies. Herein, we aimed to analyze the safety and efficacy of sintilimab plus TKI in a real-life cohort of patients with initially unresectable HCC. We present the following article in accordance with the STROBE reporting checklist (available at http://dx.doi. org/10.21037/atm-20-7037).

\section{Methods}

\section{Study design and patients}

This was a single-arm study to assess the efficacy and tolerability of sintilimab plus TKIs in patients with unresectable HCC in a real world. Patients who received sintilimab in combination with TKIs synchronously or metachronously to treat unresectable HCC in Zhongshan Hospital of Fudan University between February 2019 and December 2019 were enrolled. The study was conducted in accordance with the Declaration of Helsinki (as revised in 2013). The study was approved by institutional committee board of Zhongshan Hospital, Fudan University (No. B2019-296R) and individual consent for this retrospective analysis was waived. The eligibility criteria included: (I) unresectable HCC confirmed histologically or radiologically based on the AASLD criteria; (II) ChildPugh class A or B liver function; (III) Eastern Cooperative Oncology Group performance status 0-1; (IV) at least one measurable tumor lesion as defined by Response Evaluation Criteria in Solid Tumors, version 1.1 (RECIST 1.1); and (V) at least one follow-up imaging assessment. The exclusion criteria included: (I) history of other malignancies; (II) active hepatitis $\mathrm{B}$ with abnormal liver function (alanine aminotransferase and aspartate transaminase $\geq 5$ upper limit of normal value). All the data was censored on September $30^{\text {st }} 2020$.

\section{Treatment protocol}

Patients were assigned to receive sintilimab plus TKIs 
based on previous treatments and economic consideration. Sintilimab was prescribed at a fixed dose of $200 \mathrm{mg}$ every 3 weeks. Four available TKIs including sorafenib, lenvatinib, regorafenib, apatinib were administered orally. Sorafenib was given $400 \mathrm{mg} /$ day initially and increased to $800 \mathrm{mg} /$ day in a stepwise manner if tolerated. The lenvatinib dose was determined according to body weight as follows: patients weighing $<60 \mathrm{~kg}$ received $8 \mathrm{mg} /$ day, whereas those weighing $\geq 60 \mathrm{~kg}$ received $12 \mathrm{mg} /$ day. Regorafenib was prescribed $160 \mathrm{mg} /$ day during weeks $1-3$ of each 4 week-cycle. Apatinib was prescribed $500 \mathrm{mg} /$ day initially and increased to $750 \mathrm{mg} / \mathrm{d}$ if tolerated. Patients who experienced adverse events (AEs) due to TKIs had dose reduction, interruption or discontinuation according to the grade of toxicity. Sintilimab was interrupted or discontinued, and immunesuppressant agents were given based on the severity and the affected organs, if immunotherapy-related SAEs (irSAEs) occurred. TACE was performed before or after the systemic combination therapies in select patients with multinodular HCC or locally-advanced HCC with portal vein tumor thrombus (PVTT). Resection was performed after successful downstaging with adequate future liver remnant (FLR). Patients could continue the combination or adopt single-agent treatment beyond disease progression if the investigators observed evidence of clinical benefit.

\section{Response and safety evaluation}

Radiological response was recorded by dynamic computed tomography (CT) or magnetic resonance imaging (MRI) at baseline and every 6-12 weeks after treatment initiation. Tumor responses including ORR, disease control rate (DCR) were assessed according to the Response Evaluation Criteria in Solid Tumors (RECIST) 1.1 and HCC-specific modified RECIST (mRECIST) (17). ORR and DCR were determined as the proportion of patients with a confirmed objective response (OR) including completed response (CR) and partial response (PR), and the proportion of patients with at least one radiologically confirmed OR stable disease (SD) during the treatment respectively. PFS referred to the time from the initial dose to the first radiologically confirmed PD or death from any cause. In addition to overall response, organspecific responses were also evaluated for each affected organ. Safety was continuously evaluated by vital signs and clinical laboratory tests. AEs were evaluated according to National Cancer Institute Common Terminology Criteria for Adverse Events version 4.0.

\section{Statistical analyses}

Continuous variables were presented as median (range) or mean \pm standard deviation, and compared using $\mathrm{T}$ test or Mann-Whitney $U$ test according to the normality and homogeneity. Categorical variables were shown as frequency (percentage) and compared using chi-square test or Fisher's exact test when appropriate. Statistical analyses were performed using SPSS 22.0 software (RRID:SCR_002865) and R 4.0.2 (RRID:SCR_001905). The PFS and OS were all estimated by the Kaplan-Meier method. The survival curve was depicted using the "survminer" and "survival" packages. A propensity score was constructed to account for potential bias between the groups who had TACE combined with the systemic therapies and the groups who received systemic therapies alone. The 1:1 score matching was performed using the "MatchIt" package. Two-sided $\mathrm{P}$ values $<0.05$ was considered statistically significant.

\section{Results}

\section{Patients characteristics}

A total of 60 patients with unresectable HCC who received sintilimab plus TKIs between February 2019 and December 2019 were included in this retrospective analysis. The baseline patient characteristics are listed in Table 1. The majority of patients had HBV-associated HCC and $10.0 \%$ of patients had Child-Pugh class B liver function reserve. A total of $43(71.7 \%)$ patients were at BCLC stage C and the other 17 (28.3\%) patients had BCLC B HCC. The median size of the baseline target lesions was $99.4 \mathrm{~mm}$ (range, $10.0-170.0 \mathrm{~mm}$ ). The combination therapy was adopted as systemic first-, second-, third, or fourth-line treatment in 44 (73.3\%), 9 (15.0\%), $6(10.0 \%)$ and $1(1.7 \%)$ patients respectively. A total of 53 patients received PD-1 inhibitors and TKI synchronously. The other 7 patients received PD-1 inhibitors in addition to the prior TKIs on tumor progression. The median interval between the administration of TKIs and sequential PD-1 inhibitors was $3.8(1.8-14.2)$ months.

\section{Tumor response and safety}

As of the data cutoff on September $30^{\text {st }}, 2020$, the median duration of follow-up was 10.4 (4.3-23.9) months. A total of 13 (21.7\%) patients died. The median OS has not reached (Figure 1A). The median PFS was 8.9 months (95\% CI: 
Table 1 Baseline patient characteristics

\begin{tabular}{|c|c|}
\hline Variables & Patients $(n=60)$ \\
\hline \multicolumn{2}{|l|}{ Age } \\
\hline$\leq 60$ & $40(66.7 \%)$ \\
\hline$>60$ & $20(33.3 \%)$ \\
\hline \multicolumn{2}{|l|}{ Sex } \\
\hline Male & $51(85.0 \%)$ \\
\hline Female & $9(15.0 \%)$ \\
\hline \multicolumn{2}{|l|}{ Aetiology } \\
\hline HBV & $58(96.7 \%)$ \\
\hline Non-HBV non-HCV & $2(3.3 \%)$ \\
\hline \multicolumn{2}{|l|}{ Child-Pugh class } \\
\hline A & $54(90.0 \%)$ \\
\hline$B$ & $6(10.0 \%)$ \\
\hline \multicolumn{2}{|l|}{ HBV-DNA, IU/mL } \\
\hline$\geq 1,000$ & $11(18.3 \%)$ \\
\hline$<1,000$ & $49(81.7 \%)$ \\
\hline \multicolumn{2}{|l|}{ AFP, $\mathrm{ng} / \mathrm{mL}$} \\
\hline$\geq 400$ & $33(55.0 \%)$ \\
\hline$<400$ & $27(45.0 \%)$ \\
\hline \multicolumn{2}{|l|}{ BCLC stage } \\
\hline $\mathrm{B}$ & $17(28.3 \%)$ \\
\hline C & $43(71.7 \%)$ \\
\hline \multicolumn{2}{|l|}{ Disease sites } \\
\hline Liver & $51(85.0 \%)$ \\
\hline Liver without macrovascular invasion & $25(41.7 \%)$ \\
\hline Liver with major vascular invasion & $26(43.3 \%)$ \\
\hline Lung & $11(18.3 \%)$ \\
\hline Lymph nodes & $10(16.7 \%)$ \\
\hline Intra-abdomen & $8(13.3 \%)$ \\
\hline Bone & $4(6.7 \%)$ \\
\hline Brain & $1(1.7 \%)$ \\
\hline \multicolumn{2}{|l|}{ Combination strategies } \\
\hline Sintilimab +Lenvatinib & $48(80.0 \%)$ \\
\hline Sintilimab + Sorafenib & $3(5.0 \%)$ \\
\hline Sintilimab + Regorafenib & $4(6.7 \%)$ \\
\hline Sintilimab + Apatinib & $5(8.3 \%)$ \\
\hline
\end{tabular}

Table 1 (continued)
Table 1 (continued)

\begin{tabular}{lc}
\hline Variables & Patients $(\mathrm{n}=60)$ \\
\hline Combination therapy as systemic & \\
First-line & $44(73.3 \%)$ \\
Second-line & $9(15.0 \%)$ \\
Third-line & $6(10.0 \%)$ \\
Fourth-line & $1(1.7 \%)$ \\
\hline
\end{tabular}

HBV, hepatitis B virus; HCV, hepatitis C virus; AFP, alpha fetal protein; BCLC, Barcelona Clinic Liver Cancer.

6.9-11.0, Figure 1A). Overall, 21 (35.0\%) patients remained on the combinational therapy and $15(25.0 \%)$ patients showed ongoing tumor responses. The ORR and DCR were $36.7 \%$ (95\% CI: $24.9-48.5 \%$ ), $81.7 \%$ (95\% CI: $71.9-$ $91.5 \%$ ) according to the RECIST 1.1, and 52.8\% (95\% CI: $39.1-66.5 \%), 83.0 \%$ (95\% CI: 73.2-93.8\%) according to mRECIST criteria, respectively. The best overall responses were $20 \mathrm{PR}$ and $2 \mathrm{CR}$. The ORR ( $41.3 \%$ vs. $21.4 \%, \mathrm{P}=0.18$ ) and PFS (median, 8.6 vs. 9.1 months, $\mathrm{P}=0.77$, Figure $1 B$ ) of patients who received the combined therapies as the first line therapy were similar to those of the others who received as the second to fourth lines. Baseline tumor burden $(96.5 \pm 52.5$ vs. $76.7 \pm 45.9 \mathrm{~mm}, \mathrm{P}=0.21)$ and the percentage of BCLC C HCC (67.4\% vs. $85.7 \%, \mathrm{P}=0.32)$ were comparable between two groups. Patients who received PD-1 inhibitor sintilimab after the initiation of TKIs had similar ORR (42.9\% vs. $35.8 \%, \mathrm{P}=0.718$ ) and PFS (median, 7.4 vs. 8.9 months, $\mathrm{P}=0.68$, Figure $1 C$ ) compared to the patients who received the two systemic therapies synchronously. No significant differences in total tumor burden $(63.9 \pm 59.7$ vs. $95.5 \pm 49.6 \mathrm{~mm}, \mathrm{P}=0.13)$ and BCLC stage $\mathrm{C}$ percentage (71.4\% vs. $71.7 \%, \mathrm{P}=0.99)$ were observed between patients treated with PD-1 inhibitor and TKI metachronously and those synchronously.

Regarding organ-specific response, 51, 11, 10 and 8 patients had measurable hepatic, lung, lymph node and other intra-abdominal metastases at baseline respectively. The intra-abdominal lesions included peritoneal, adrenal and splenic metastases. The median sizes of hepatic tumors and lung, lymph node and intra-abdominal were $92.0 \mathrm{~mm}$ (10.0-170.0), $13.1 \mathrm{~mm}$ (10.2-57.3), $31.6 \mathrm{~mm}$ (15.0-111.6), and $42.2 \mathrm{~mm}$ (16.6-48.2) respectively. Organ-specific ORRs of hepatic tumors and lung, lymph node, intra-abdominal metastases were $35.3 \%, 16.7 \%, 40.0 \%$, and $25.0 \%$, respectively. Best reductions from baseline in organ-specific tumor burden are shown in Figure 2A. One patient who 

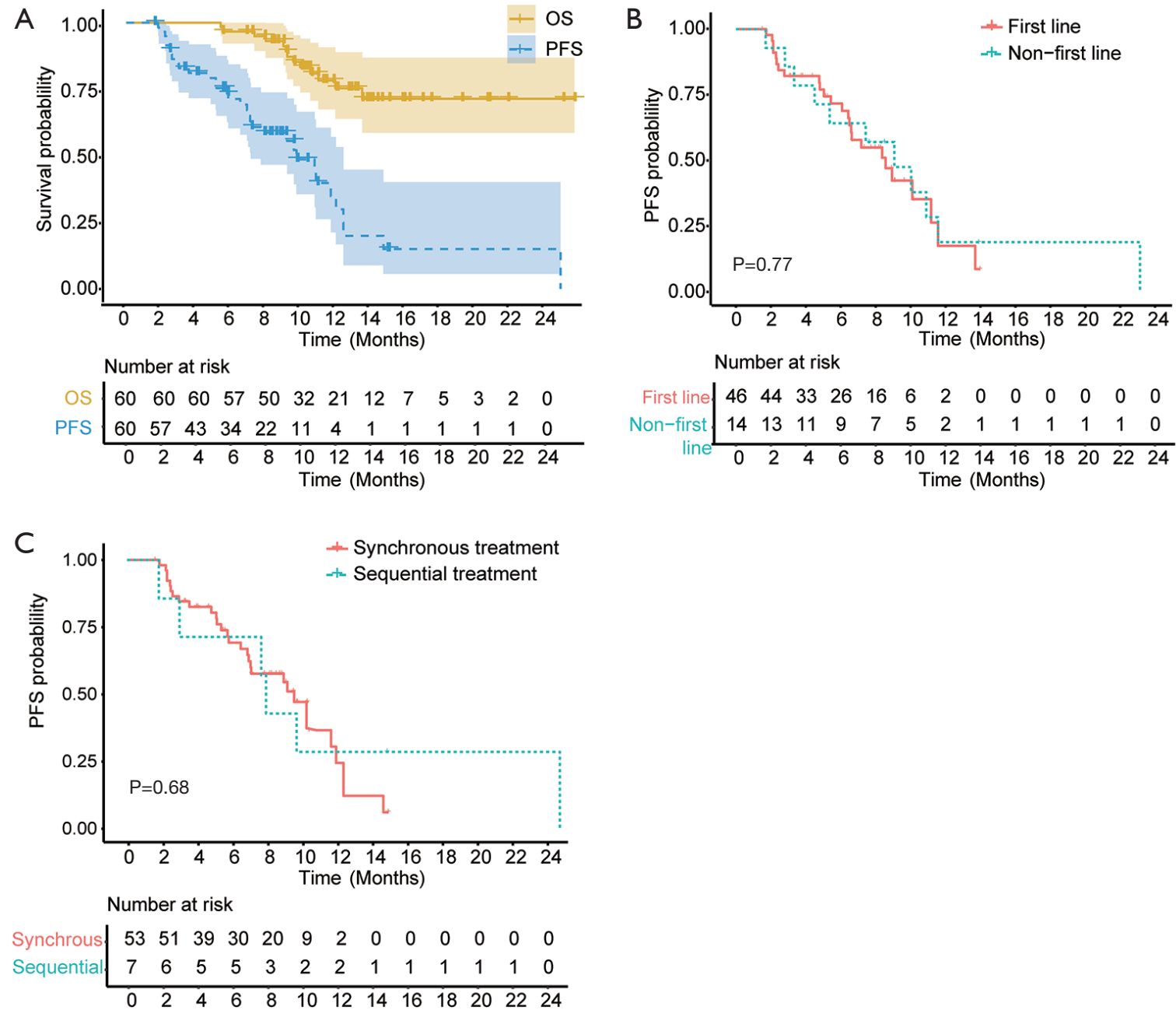

Figure 1 Kaplan-Meier estimates of progression-free survival (PFS) and overall survival (OS). (A) PFS and OS of overall patient cohort. (B) Comparison of PFS between patients receiving the combination therapy as the first line treatment and those as the second to fourth line. (C) Comparison of PFS between patients receiving sintilimab plus TKIs synchronously and those receiving sintilimab after TKIs.

had brain metastases showed PR in the brain lesions with parallel decreasing alpha fetoprotein (AFP) level after the combined therapy of lenvatinib plus 2 sessions of sintilimab (Figure 2B).

Overall, 46 (76.7\%) patients reported adverse event (AE) with any grade and $8(13.3 \%)$ patients discontinued the combination therapy due to severe adverse events (SAE). The most common AEs included fatigue, appetite loss, hypertension, hand-foot syndrome. Patients with ChildPugh B liver function had significantly higher incidence of grade 3/4 adverse events than those with Child-Pugh A diseases $(83.3 \%$ vs. $5.6 \%, \mathrm{P}<0.01)$. Grade 4 skin toxicity was observed in 1 patient after the treatment of 3 cycles of sintilimab plus apatinib, and the patient recovered from discontinuation of sintilimab and treatment of prednisone. Immune-related myocarditis and thyroiditis was identified in one patient with elevated myocardial enzyme and abnormal thyroid function after lenvatinib and 2 cycles of sintilimab. The cardiac MRI confirmed myocardial damage. The patient recovered from concurrent myocarditis and thyroiditis after prednisone treatment for nearly 2 months. And dynamic MRI confirmed sustained CR after discontinuation of sintilimab. Two patients had grade 4 liver function impairment. Liver biopsy confirmed immunerelated hepatitis in one patient and cholestasis without immune-related evidence in the other one. Four of the six patients with mild to moderate ascites due to cirrhosis at baseline had significant increased ascites after the combined 

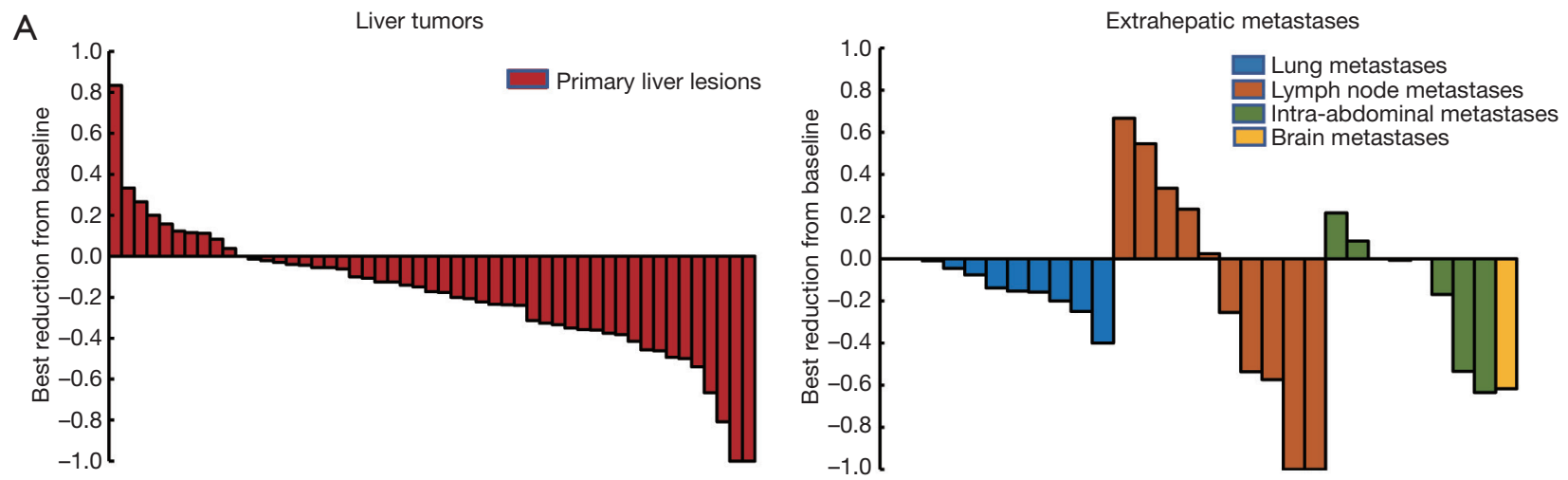

B
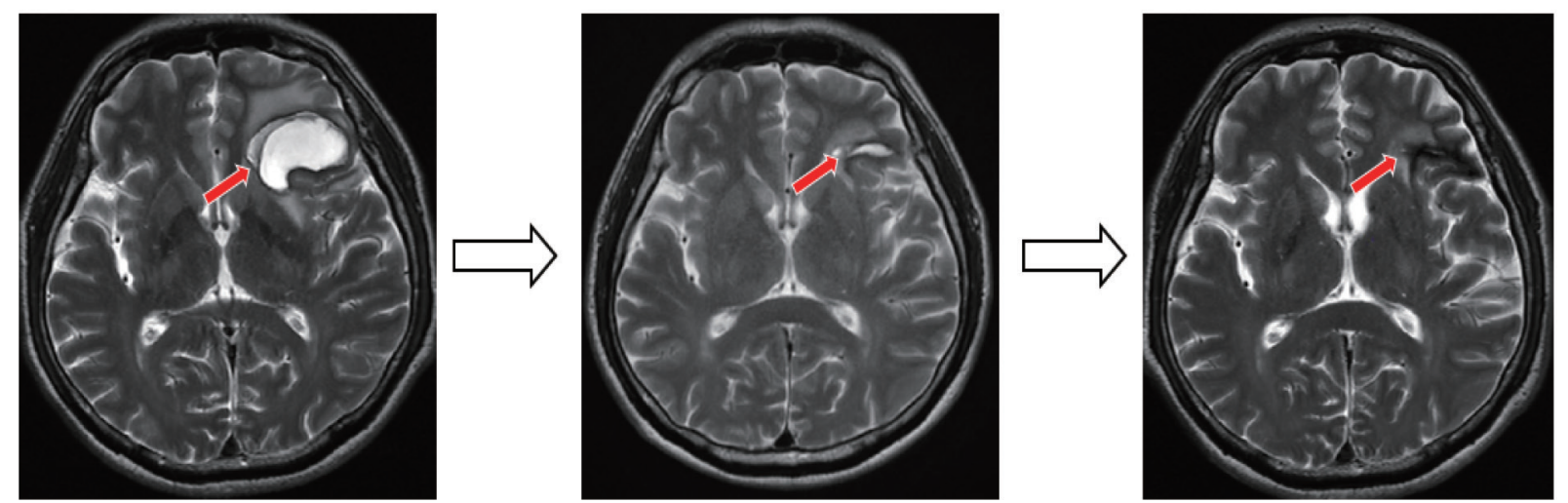

Figure 2 Organ-specific tumor responses. (A) Best reduction from baseline in liver tumors and extrahepatic metastases. (B) Representative images showing response in brain metastasis in one patient: shrinkage of brain metastasis with intra-tumor hemorrhage from baseline (left panel) to follow-ups after lenvatinib plus 2 cycles (middle panel) and 4 cycles (right panel) of sintilimab. The arrows indicate brain metastasis.

systemic therapies for 2 to 4 months, including 2 patients had upper gastrointestinal hemorrhage. The combined systemic therapies were discontinued, although the imaging evaluation showed PR or SD in the liver tumors and the laboratory results showed no significant change in liver function. Patients with HBV-DNA $\geq 1,000 \mathrm{IU} / \mathrm{mL}$ had similar incidence of liver-related adverse events to those with HBV-DAN <1,000 IU/mL (27.3\% vs. 18.4\%, P=0.80).

\section{Local regional therapies plus the combined systemic therapy}

Among 36 HCC patients with multinodular HCC or locally-advanced HCC with portal vein tumor thrombus (PVTT), 11 patients received TACE approximately 1 week to 1 month before the combination systemic therapies and 3 patients received TACE within 1 month after the first dose of PD-1 inhibitor. The rest 22 patients did not receive any LRT. After 1:1 propensity score matching, 24 patients were matched. Patients from the TACE group and non-TACE group had the same proportion of patients aged $>60$ years (25\%) and male (83.3\%), Child-Pugh B (8.3\%) and BCLC $\mathrm{C}(50 \%)$ patients. Patients from the TACE group tended to a longer PFS (median, 10.1 vs. 9.1 months, $\mathrm{P}=0.73$, Figure 3) than those from the non-TACE group but without significant differences. The ORR ( $50 \%$ vs. $66.7 \%, \mathrm{P}=0.41)$ and the incidences of SAEs ( $37.5 \%$ vs. $15.4 \%, \mathrm{P}=0.33)$ were comparable between two groups.

\section{Surgical resection after the combined systemic therapy}

A total of 8 patients received surgical resections after the combined systemic therapy. Overall, 7 patients had successful resection and the other 1 patient died of immune-related hepatitis and colitis after resection. The median tumor diameter was $130.0 \mathrm{~mm}(92.0-170.0 \mathrm{~mm})$ at baseline and decreased to $85.0 \mathrm{~mm}(50.2-130.3 \mathrm{~mm})$ before resection. Notably, 4 patients had PR after 2 to 8 cycles of sintilimab plus TKIs, and resections were 
A
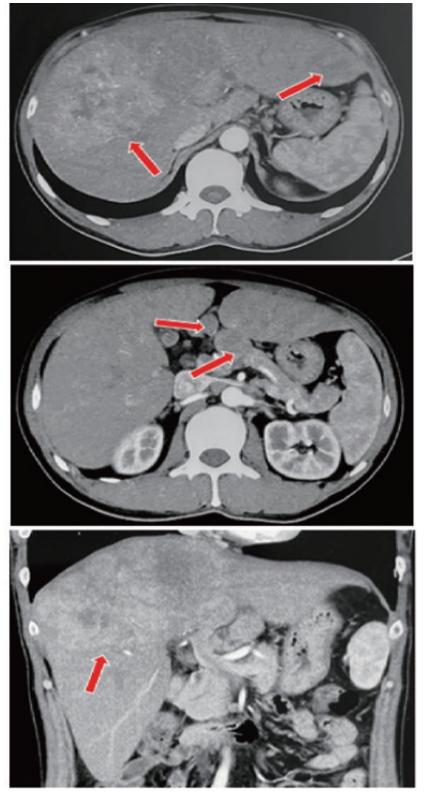

B

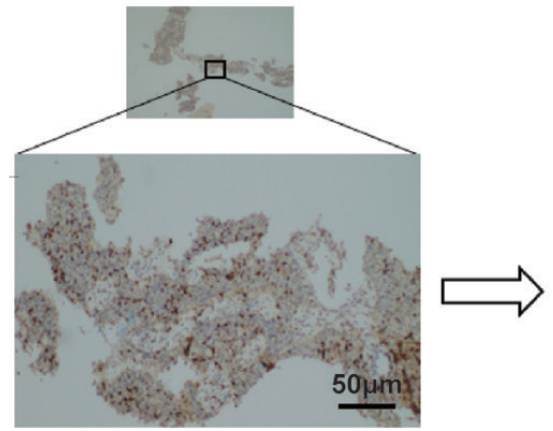

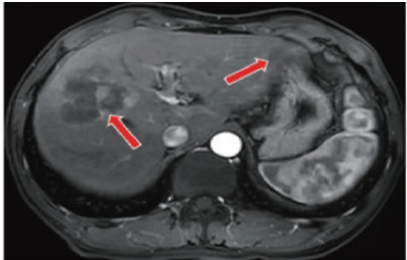
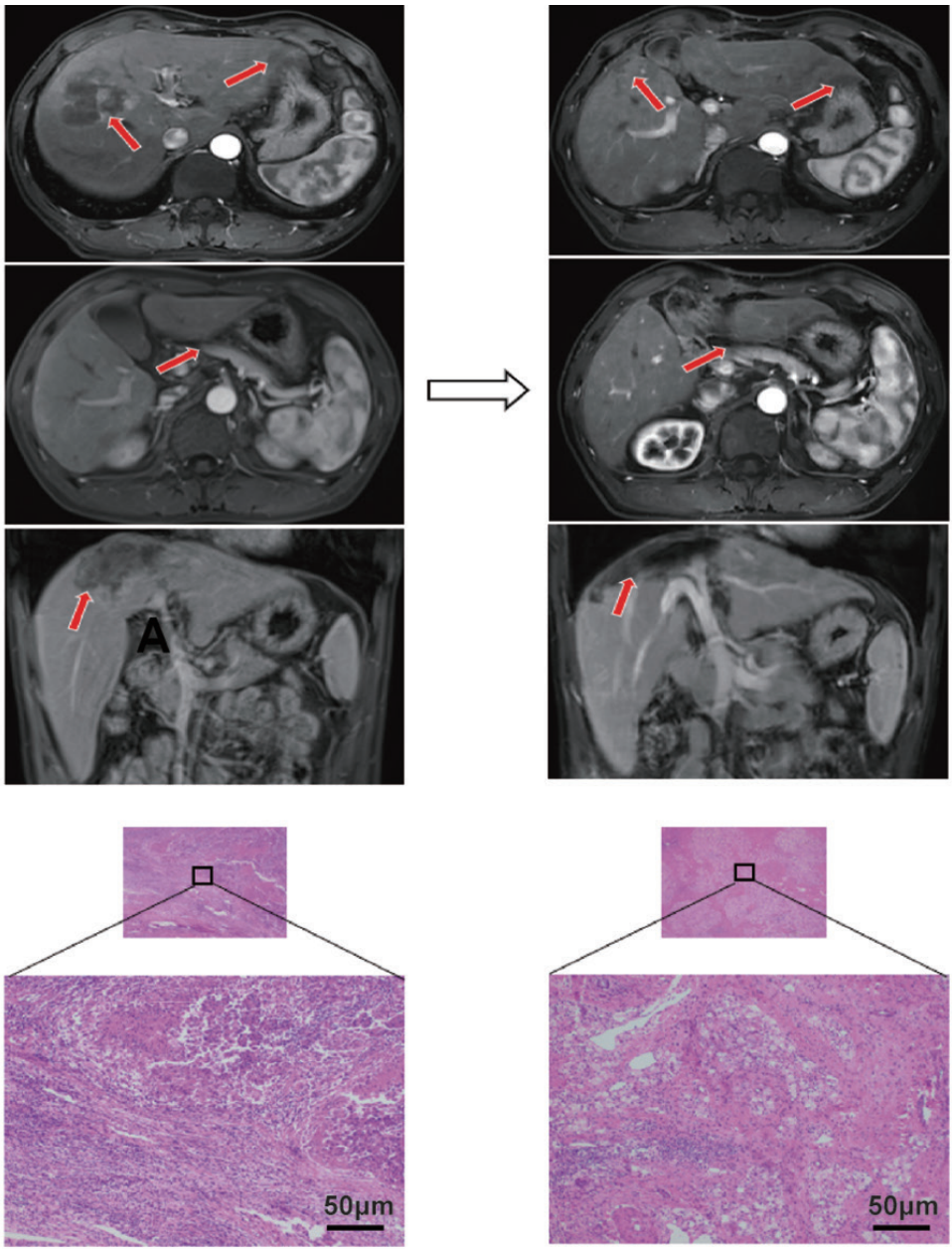

Figure 3 Representative images showing successful resection after downstaging induced by sintilimab plus TKI in one patient. (A) Change of liver lesions and lymph node metastases after the combined treatment: diffuse liver lesions in the right and left lobe and peritoneal lymph node metastases at baseline (left panel), shrinkage of liver lesions and disappearance of lymph node metastases after treatment with 5 cycles of sintilimab plus TKI (middle panel), no intrahepatic liver recurrence or peritoneal metastasis 1 month after resection (right panel). The arrows indicate intrahepatic lesions and lymph node metastases. (B) Pathological results of the biopsy at baseline and the resected lesions after the combination therapies: PD-1 staining positive in $30 \%$ of the biopsy lesions before the combination treatment (left panel), residual viable tumor with necrosis, diffuse inflammatory cells infiltration and interstitial fibrosis (middle panel) as well as histiocytic reaction (right panel) in the resected lesions.

performed approximately 1 week to 1 month after the last administration of sintilimab. All the 4 patients exhibited massive HCC with PVTT at baseline, including 3 patients with $\mathrm{pV} 3$ and the other 1 with $\mathrm{pV} 4$ PVTT. Two of the four patients with abdominal lymph node invasion at baseline showed disappearance of the metastases upon the first imaging evaluation after the combined treatments. The pathological results of the resected lesions and extracted PVTT showed complete necrosis with immunocytes infiltration and fibroplastic proliferation in 3 patients, and remnant viable tumor in $30 \%$ of the resected lesions in the other patient (Figure 4). The fifth patient had severe erythema multiforma after the combined treatment of levatinib plus 3 cycles of sintilimab. Resections were performed upon metastasis to the right adrenaline while the liver lesion exhibited continuous PR for 4 months after discontinuation of sintilimab. Interestingly, the pathological result showed mild residual viable tumor in the liver lesion and no viable tumor but medullary hyperplasia in the right adrenaline although both dynamic MRI and PET-CT showed active tumor signs. No recurrence or metastasis was observed 3.4-13.0 months post resection in 


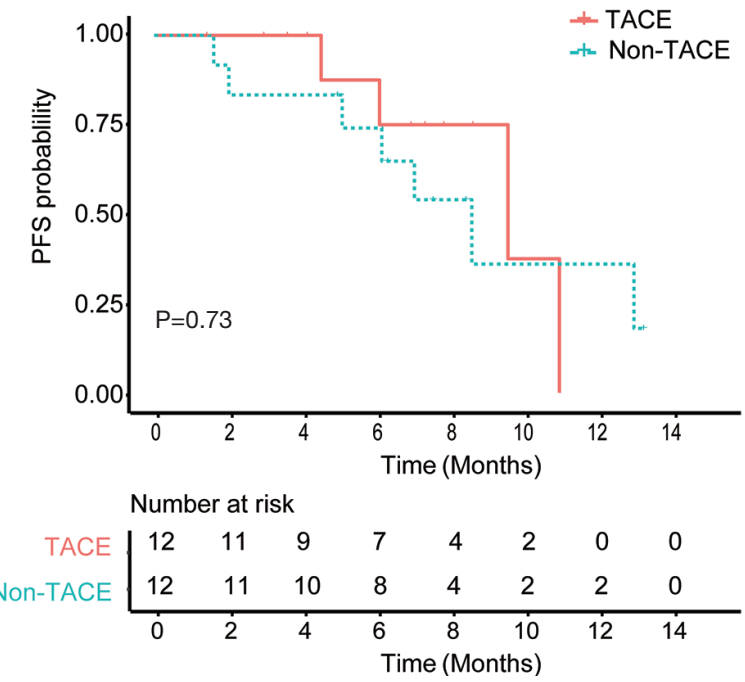

Figure 4 Comparison of PFS between treatment-naive patients who had TACE in addition to the combined systemic therapies and those who did not have TACE treatment.

the above five patients and the sixth patient with BCLC B HCC responding to the combined sintilimab with lenvatinib treatment. Another patient refused to receive resection until satellite nodules appeared around the already shrunk targeted lesion. The pathological result after resection exhibited viable tumor in both the target lesion and surrounding satellite nodules without significant accumulation of immunocytes. Intrahepatic recurrence occurred 1 month after the resection and TACE was performed subsequently. The last patient had dissection of the liver lesion and metastatic portal lymph node after the treatment of lenvatinib plus 5 cycles of sintilimab, and died of immune-related hepatitis and colitis in the perioperative period.

\section{Discussion}

As a chance for cure, PD-1 inhibitors have revolutionized cancer treatment. The modulatory effect of TKIs targeting VEGFRs and other kinases on tumor microenvironment tend to enhance the therapeutic effect of PD-1 inhibitors, and vice versa (18). The synergistic effect of TKIs plus PD-1 inhibitors have been demonstrated in many cancers (12). We demonstrated that PD-1 inhibitors plus TKIs showed promising therapeutic effect in a real-world cohort of patients with unresectable HCC. The ORR of TKIs plus PD-1 inhibitors reached $36.7 \%$ in our cohort, higher than that of PD-1 inhibitor or TKIs monotherapy in previous studies (3-10). The ORRs of lenvatinib plus nivolumab, lenvatinib plus pembrolizumab were $54.1 \%$ and $36.7 \%$ respectively in two phase Ib studies, the results of which were similar to that in our study $(13,19)$. While clinical trials only included Child-Pugh A patients to minimize the confounding effect of liver failure on overall outcome, our studies also included patients with Child-Pugh B patients. The grade $3 / 4$ adverse events were significantly higher in the Child-Pugh B patients than that in the Child-Pugh A patients. Although PD-1 inhibitors are demonstrated to be safe in patients with Child-Pugh B or C liver function reserve in real-world practices, the addition of TKIs should be evaluated more carefully $(20,21)$. On the other hand, the combination therapies are shown to be safe for patients with high viral burden and can be administered concurrently with anti-viral agents, the safety of which needs further investigation in a large population as HBV-DNA is usually required to be controlled $\leq 1,000 \mathrm{IU} / \mathrm{mL}$ in the clinical trials.

Neoadjuvant PD-1 blockade by enhancing both the local and systemic antitumor immune response is suggested as an efficacious approach in resectable lung cancer or recurrent glioblatoma $(22,23)$. Its counterpart efficacy in HCC has been reported with pCR reaching $24 \%$ (24). Here we reported that PD-1 inhibitor plus TKI offered patients with otherwise unresectable HCC a chance for curative surgery by downstaging of huge HCC and by alleviation of PVTT and even extrahepatic metastases. These conversion cases are indicative of another effective attempt for advanced HCC treatment. Although 3 of the 8 patients turned out to be pathological CR in our study, the tumor resection and PVTT extraction resulted in better liver function by introduction of the remnant liver hypertrophy and by alleviation of portal vein hypertension. Compared to traditional FLR augmentation by portal vein embolization (PVE) or associating liver partition and portal vein ligation for staged hepatectomy (ALPPS) (25), the combined systemic therapies are applicable for more advanced HCC in the responders, especially for those with extrahepatic metastases. Immune-related SAE should be paid special attention and pre-surgery liver biopsy was performed in some candidates in our center. Currently, patients with active immune cell infiltration with moderate to severe inflammation in the normal liver tissue have been excluded from tumor resection, which needs to be testified in a large population. Overall, surgical resection after response to the combined systemic therapies turned out to be safe without recurrence in the short term. Besides the long-term prognosis after resection, another problem to be focused 
on is whether PD-1 blockade or TKI, alone or combined, should be continued as adjuvant setting after resection.

It has been noted for years that LRTs play a significant role in systemic immunomodulation other than simple tumor elimination. Ablation and TACE cause immunogenic cell death, leading to the release of various tumor antigens and immune activation. Studies have been conducted to test the hypothesis that immune checkpoint blockade may enhance immune responses caused by LRTs (NCT03572582, NCT03397654, NCT03383458, NCT03143270). Duffy et al. treated 39 patients with LRTs (ablation or DEB-TACE) plus anti-CTLA4 tremelimumab and reported an ORR of $23.5 \%$ with tolerable toxicity among 34 evaluable patients (26). Furthermore, TKI combination might prevent the TACE-induced surge in proangiogenic factors and reduce tumor volume by normalization of tumor vessels. Despite three negative clinical studies, the TACTICS trial demonstrated that pretreatment with sorafenib 2-3 weeks before initial TACE procedure and continued use of sorafenib after TACE significantly improved PFS in patients with unresectable HCC (27-30). One of the trial design differences lies in that the sorafenib pretreatment duration was 3-7 days and 2-3 weeks in the SPACE and the TACTICS trial respectively $(27,28)$. In our small-sample study, TACE in addition to the combination systemic therapies of TKI and PD-1 inhibitor exhibited a tendency of improved PFS in a more advanced-stage population. As we performed TACE procedure within 1 month before or after the combined systemic therapies, whether different sequence and time interval of TKI, PD-1 inhibitor and TACE administration will elicit different tumor response and liver function reserve is another issue that needs to be addressed.

The heterogenous tumor microenvironments and the distinct proteogenomic characterization of various tumors of different patients influence the responses to anticancer treatments in HCC (31-34). While various molecular markers predicting the therapeutic efficacy of TKI or PD-1 inhibitors are identified in the tissue or in the peripheral blood, how to integrate these markers to optimize treatments in a clinical setting is a hotspot issue to be addressed. Differential organ-specific tumor response was observed in many solid tumors $(35,36)$. Lung metastases exhibited the best response to ICIs in a previous real-world cohort of patients with advanced HCC (37). Consistently, lung metastases also revealed favorable response to PD-1 inhibitors plus TKIs in our study. We also observed brain metastasis response in one patient. The efficacy of pembrolizumab in brain metastases has been demonstrated in patients with melanoma or non-small cell lung cancer (38), while patients diagnosed with HCC and brain metastasis are always excluded from clinical trials. The efficacy of PD-1 inhibitor plus TKI in HCC patients with brain metastases needs to be testified in a large population.

The study had several limitations. First of all, the relative small sample reduced the statistic power. As the majority of patients had HBV infection, the result might not be applicable for HCC of other etiologies. Moreover, the intervention of TACE was not randomized, controlled design and selection bias could not be avoided.

\section{Conclusions}

PD-1-targeted immunotherapy plus molecular-targeted therapy exhibited promising efficacy with tolerable toxicity in unresectable HCC in the real word. For select responders, surgical resection might be a choice for radical treatment. And the addition of TACE to the combined systemic therapies also resulted in a favorable tumor control and safety. Identification of biomarkers to select patients most likely to benefit from different treatment modalities and translation of the molecular findings to the clinical setting should be highlighted in HCC.

\section{Acknowledgments}

Funding: This work was sponsored by Program of Shanghai Academic Research Leader [19XD1420700] and supported by National Natural Science Foundation of China [91859105, 8196112802]; the Basic Research Project from Technology Commission of Shanghai Municipality and the Shanghai Municipal Key Clinical Specialty [17JC1402200].

\section{Footnote}

Reporting Checklist: The authors have completed the STROBE reporting checklist. Available at http://dx.doi. org/10.21037/atm-20-7037

Data Sharing Statement: Available at http://dx.doi. org/10.21037/atm-20-7037

Conflicts of Interest: All authors have completed the ICMJE uniform disclosure form (available at http://dx.doi. org/10.21037/atm-20-7037). The authors have no conflicts of interest to declare. 
Ethical Statement: The authors are accountable for all aspects of the work in ensuring that questions related to the accuracy or integrity of any part of the work are appropriately investigated and resolved. The study was conducted in accordance with the Declaration of Helsinki (as revised in 2013). The study was approved by institutional committee board of Zhongshan Hospital, Fudan University (NO. B2019-296R) and individual consent for this retrospective analysis was waived.

Open Access Statement: This is an Open Access article distributed in accordance with the Creative Commons Attribution-NonCommercial-NoDerivs 4.0 International License (CC BY-NC-ND 4.0), which permits the noncommercial replication and distribution of the article with the strict proviso that no changes or edits are made and the original work is properly cited (including links to both the formal publication through the relevant DOI and the license). See: https://creativecommons.org/licenses/by-nc-nd/4.0/.

\section{References}

1. Siegel RL, Miller KD, Jemal A. Cancer statistics, 2019. CA Cancer J Clin 2019;69:7-34.

2. Llovet JM, Zucman-Rossi J, Pikarsky E, et al. Hepatocellular carcinoma. Nat Rev Dis Primers 2016;2:16018.

3. Llovet JM, Ricci S, Mazzaferro V, et al. Sorafenib in advanced hepatocellular carcinoma. $\mathrm{N}$ Engl J Med 2008;359:378-90.

4. Kudo M, Finn RS, Qin S, et al. Lenvatinib versus sorafenib in first-line treatment of patients with unresectable hepatocellular carcinoma: a randomised phase 3 noninferiority trial. Lancet 2018;391:1163-73.

5. Finn RS, Merle P, Granito A, et al. Outcomes of sequential treatment with sorafenib followed by regorafenib for HCC: Additional analyses from the phase III RESORCE trial. J Hepatol 2018;69:353-8.

6. Abou-Alfa GK, Meyer T, Cheng AL, et al. Cabozantinib in Patients with Advanced and Progressing Hepatocellular Carcinoma. N Engl J Med 2018;379:54-63.

7. Zhu AX, Kang YK, Yen CJ, et al. Ramucirumab after sorafenib in patients with advanced hepatocellular carcinoma and increased alpha-fetoprotein concentrations (REACH-2): a randomised, double-blind, placebocontrolled, phase 3 trial. Lancet Oncol 2019;20:282-96.

8. Li Q, Qin SK, Gu ZS, et al. Apatinib as second-line therapy in Chinese patients with advanced hepatocellular carcinoma: A randomized, placebo-controlled, doubleblind, phase III study. J Clin Oncol 2020;38:4507.

9. Zhu AX, Finn RS, Edeline J, et al. Pembrolizumab in patients with advanced hepatocellular carcinoma previously treated with sorafenib (KEYNOTE-224): a non-randomised, open-label phase 2 trial. Lancet Oncol 2018;19:940-52.

10. El-Khoueiry AB, Sangro B, Yau T, et al. Nivolumab in patients with advanced hepatocellular carcinoma (CheckMate 040): an open-label, non-comparative, phase $1 / 2$ dose escalation and expansion trial. Lancet 2017;389:2492-502.

11. Finn RS, Ryoo BY, Merle P, et al. Pembrolizumab As Second-Line Therapy in Patients With Advanced Hepatocellular Carcinoma in KEYNOTE-240: A Randomized, Double-Blind, Phase III Trial. J Clin Oncol 2020;38:193-202.

12. Taylor MH, Lee CH, Makker V, et al. Phase IB/II Trial of Lenvatinib Plus Pembrolizumab in Patients With Advanced Renal Cell Carcinoma, Endometrial Cancer, and Other Selected Advanced Solid Tumors. J Clin Oncol 2020;38:1154-63.

13. Zhu AX, Finn RS, Ikeda M, et al. A phase Ib study of lenvatinib (LEN) plus pembrolizumab (PEMBRO) in unresectable hepatocellular carcinoma (uHCC). J Clin Oncol 2020;38:4519.

14. Finn RS, Qin S, Ikeda M, et al. Atezolizumab plus Bevacizumab in Unresectable Hepatocellular Carcinoma. N Engl J Med 2020;382:1894-905.

15. Wang J, Fei K, Jing H, et al. Durable blockade of PD-1 signaling links preclinical efficacy of sintilimab to its clinical benefit. MAbs 2019;11:1443-51.

16. Zhang W, Bi XY, Sun YK, et al. Preliminary results of sintilimab plus different dose of IBI305 (anti-VEGF) monoclonal antibody) in patients with advanced hepatocellular carcinoma: A phase Ib study. J Clin Oncol 2020;38:abstr 3079.

17. Lencioni R, Llovet JM. Modified RECIST (mRECIST) assessment for hepatocellular carcinoma. Semin Liver Dis 2010;30:52-60.

18. Jain RK. Antiangiogenesis strategies revisited: from starving tumors to alleviating hypoxia. Cancer Cell 2014;26:605-22.

19. Ikeda M, Sung MW, Kudo M, et al. A phase $1 \mathrm{~b}$ study of lenvatinib plus nivolumab in patients with unresectable hepatocellular carcinoma (Study 117). J Clin Oncol 2020;36:4076.

20. Scheiner B, Kirstein MM, Hucke F, et al. Programmed 
cell death protein-1 (PD-1)-targeted immunotherapy in advanced hepatocellular carcinoma: efficacy and safety data from an international multicentre real-world cohort. Aliment Pharmacol Ther 2019;49:1323-33.

21. Cui H, Dai G, Guan J. Programmed Cell Death Protein-1 (PD-1)-Targeted Immunotherapy for Advanced Hepatocellular Carcinoma in Real World. Onco Targets Ther 2020;13:143-9.

22. Forde PM, Chaft JE, Smith KN, et al. Neoadjuvant PD-1 Blockade in Resectable Lung Cancer. N Engl J Med 2018;378:1976-86.

23. Cloughesy TF, Mochizuki AY, Orpilla JR, et al. Neoadjuvant anti-PD-1 immunotherapy promotes a survival benefit with intratumoral and systemic immune responses in recurrent glioblastoma. Nat Med 2019;25:477-86.

24. Kaseb AO, Cao HST, Mohamed YI, et al. Final results of a randomized, open label, perioperative phase II study evaluating nivolumab alone or nivolumab plus ipilimumab in patients with resectable HCC. J Clin Oncol 38: 2020 (suppl; abstr 4599). DOI:10.1200/JCO.2020.38.15_ suppl.4599.

25. Chan A, Zhang WY, Chok K, et al. ALPPS Versus Portal Vein Embolization for Hepatitis-related Hepatocellular Carcinoma: A Changing Paradigm in Modulation of Future Liver Remnant Before Major Hepatectomy. Ann Surg 2021;273:957-65.

26. Duffy AG, Ulahannan SV, Makorova-Rusher O, et al. Tremelimumab in combination with ablation in patients with advanced hepatocellular carcinoma. J Hepatol 2017;66:545-51.

27. Kudo M, Ueshima K, Ikeda M, et al. Randomised, multicentre prospective trial of transarterial chemoembolisation (TACE) plus sorafenib as compared with TACE alone in patients with hepatocellular carcinoma: TACTICS trial. Gut 2020;69:1492-501.

28. Lencioni R, Llovet JM, Han G, et al. Sorafenib or placebo plus TACE with doxorubicin-eluting beads for intermediate stage HCC: The SPACE trial. J Hepatol 2016;64:1090-8.

Cite this article as: Xie D, Sun Q, Wang X, Zhou J, Fan J, Ren Z, Gao Q. Immune checkpoint inhibitor plus tyrosine kinase inhibitor for unresectable hepatocellular carcinoma in the real world. Ann Transl Med 2021;9(8):652. doi: 10.21037/atm20-7037
29. Kudo M, Imanaka K, Chida N, et al. Phase III study of sorafenib after transarterial chemoembolisation in Japanese and Korean patients with unresectable hepatocellular carcinoma. Eur J Cancer 2011;47:2117-27.

30. Meyer T, Fox R, Ma Y'T, et al. Sorafenib in combination with transarterial chemoembolisation in patients with unresectable hepatocellular carcinoma (TACE 2): a randomised placebo-controlled, double-blind, phase 3 trial. Lancet Gastroenterol Hepatol 2017;2:565-75.

31. Kurebayashi Y, Ojima H, Tsujikawa H, et al. Landscape of immune microenvironment in hepatocellular carcinoma and its additional impact on histological and molecular classification. Hepatology 2018;68:1025-41.

32. Gao Q, Zhu H, Dong L, et al. Integrated Proteogenomic Characterization of HBV-Related Hepatocellular Carcinoma. Cell 2019;179:561-77.e22.

33. Cancer Genome Atlas Research Network. Electronic address wbe, Cancer Genome Atlas Research N. Comprehensive and Integrative Genomic Characterization of Hepatocellular Carcinoma. Cell 2017;169:1327-41.e23.

34. Dong LQ, Peng LH, Ma LJ, et al. Heterogeneous immunogenomic features and distinct escape mechanisms in multifocal hepatocellular carcinoma. J Hepatol 2019. [Epub ahead of print]. DOI: 10.1016/j.jhep.2019.12.014.

35. van Kessel CS, Samim M, Koopman M, et al. Radiological heterogeneity in response to chemotherapy is associated with poor survival in patients with colorectal liver metastases. Eur J Cancer 2013;49:2486-93.

36. Chen ZY, Zhong WZ, Zhang XC, et al. EGFR mutation heterogeneity and the mixed response to EGFR tyrosine kinase inhibitors of lung adenocarcinomas. J Hepatol 2020;72:896-908.

37. Lu LC, Hsu C, Shao YY, et al. Differential Organ-Specific Tumor Response to Immune Checkpoint Inhibitors in Hepatocellular Carcinoma. Liver Cancer 2019;8:480-90.

38. Goldberg SB, Gettinger SN, Mahajan A, et al.

Pembrolizumab for patients with melanoma or non-smallcell lung cancer and untreated brain metastases: early analysis of a non-randomised, open-label, phase 2 trial. Lancet Oncol 2016;17:976-83. 